\title{
Semantic Interaction for Visual Text Analytics
}

\author{
Alex Endert \\ Virginia Tech \\ Blacksburg, VA USA \\ aendert@vt.edu
}

\author{
Patrick Fiaux \\ Virginia Tech \\ Blacksburg, VA USA \\ pfiaux@vt.edu
}

\author{
Chris North \\ Virginia Tech \\ Blacksburg, VA USA \\ north@vt.edu
}

\begin{abstract}
Visual analytics emphasizes sensemaking of large, complex datasets through interactively exploring visualizations generated by statistical models. For example, dimensionality reduction methods use various similarity metrics to visualize textual document collections in a spatial metaphor, where similarities between documents are approximately represented through their relative spatial distances to each other in a 2D layout. This metaphor is designed to mimic analysts' mental models of the document collection and support their analytic processes, such as clustering similar documents together. However, in current methods, users must interact with such visualizations using controls external to the visual metaphor, such as sliders, menus, or text fields, to directly control underlying model parameters that they do not understand and that do not relate to their analytic process occurring within the visual metaphor. In this paper, we present the opportunity for a new design space for visual analytic interaction, called semantic interaction, which seeks to enable analysts to spatially interact with such models directly within the visual metaphor using interactions that derive from their analytic process, such as searching, highlighting, annotating, and repositioning documents. Further, we demonstrate how semantic interactions can be implemented using machine learning techniques in a visual analytic tool, called ForceSPIRE, for interactive analysis of textual data within a spatial visualization. Analysts can express their expert domain knowledge about the documents by simply moving them, which guides the underlying model to improve the overall layout, taking the user's feedback into account.
\end{abstract}

\section{Author Keywords}

Visualization; visual analytics; interaction

\section{ACM Classification Keywords}

H5.m. Information interfaces and presentation (e.g., HCI): Miscellaneous.

\section{General Terms \\ Design; Human Factors; Theory}

Permission to make digital or hard copies of all or part of this work for personal or classroom use is granted without fee provided that copies are not made or distributed for profit or commercial advantage and that copies bear this notice and the full citation on the first page. To copy otherwise, or republish, to post on servers or to redistribute to lists, requires prior specific permission and/or a fee.

CHI'12, May 5-10, 2012, Austin, Texas, USA.

Copyright 2012 ACM 978-1-4503-1015-4/12/05...\$10.00.

\section{INTRODUCTION}

Visual analytics bases its success on combining the abilities of statistical models, visualization, and human intuition for users to gain insight into large, complex datasets [23]. This success often hinges on the ability for users to interact with the information, manipulating the visualization based on their domain expertise, interactively exploring possible connections, and investigating hypotheses. It is through this interactive exploration that users are able to make sense of complex datasets, a process referred to as sensemaking [19].

The two primary parts of sensemaking are foraging and synthesis. Foraging refers to the stages of the process where users filter and gather collections of interesting or relevant information. Then, using that information, users advance through the synthesis stages of the process, where they construct and test hypotheses about how the foraged information may relate to the larger plot. Tools exist that support users for either foraging or synthesis - but not both.

In this paper we present semantic interaction, combining the foraging abilities of statistical models with the spatial synthesis abilities of analysts. Semantic interaction is based on the following principles:

1. Visual "near=similar" metaphor supports analysts' spatial cognition, and is generated by statistical models and similarity metrics. [22]

2. Use semantic interactions within the visual metaphor, based on common interactions occurring in spatial analytic processes [4] such as searching, highlighting, annotating, and repositioning documents.

3. Interpret and map the semantic interactions to the underlying parameters of the model, by updating weights and adding information.

4. Shield the users from the complexity of the underlying mathematical models and parameters.

5. Models learn incrementally by taking into account interaction during the entire analytic process, supporting analysts' process of incremental formalism [10].

6. Provide visual feedback of the updated model and learned parameters within the visual metaphor.

7. Reuse learned model parameters in future or streaming data within the visual metaphor.

To demonstrate the concept of semantic interaction, we present a prototype visual analytics tool, ForceSPIRE, for spatial analysis of textual information. In ForceSPIRE, the user interaction takes on a deeper, more integrated role in 
the exploratory spatial analytic process. This is done through capturing the semantic interaction, interpreting the analytical reasoning associated with the interaction, and updating the statistical model, and ultimately updating the spatialization. Hence, users are able to leverage semantic interaction to explore and analyze the data interactively, while the system is responsible for properly updating the underlying statistical model.

\section{RELATED WORK}

Foraging Tools

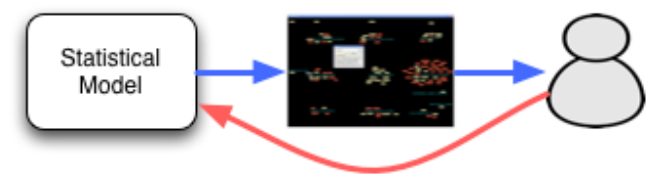

Figure 1. A model of interaction with foraging tools. Users interact directly with the statistical model (red), then gain insight through observing the change in the visualization (blue).

We categorize foraging tools by their ability to pass data through complex statistical models and visualize the computed structure of the dataset for the user to gain insight (Figure 1). Thus, users interact with these tools primarily through directly manipulating the parameters of the model used for computing the structure. As such, users are required to translate their domain expertise and semantics about the information to determine which (and by how much) to adjust these parameters. The following examples further describe this category of tools.

Visualizations such as IN-SPIRE's “Galaxy View” (shown in Figure 3) present users with a spatial layout of textual information where similar documents are proximally close to one another [25]. An algorithm creates the layout by mapping the high-dimensional collection of text documents down to a two-dimensional view. In these spatializations, the spatial metaphor is one from which users can infer meaning of the documents based on their location. The notion of distance between documents represents how similar the two documents are (i.e., more similar documents are placed closer together). For instance, a cluster of documents represents a group of similar documents, and documents placed between two clusters implies those documents are connected to both clusters. These views are beneficial as they allow users to visually gain a quick overview of the information, such as what key themes or groups exist within the dataset. The complex statistical models that compute similarity between documents are based on the structure within the data, such as term or entity frequency. In order to interactively change the view, users are required to directly adjust keyword weights, add or remove documents/keywords, or provide more information on how to parse the documents for keywords/entities upon import.
Similarly, an interactive visualization tool called iPCA uses Principal Component Analysis (PCA) to reduce highdimensional data down to a two-dimensional plot, providing users with sliders and other visual controls for directly adjusting numerous parameters of the algorithm, such as individual eigenvalues, eigenvectors, and other components of PCA [15]. Through adjusting the parameters, the user can observe how the visualization changes. This allows users to gain insight into a dataset, given they have a thorough understanding of PCA, necessary to understand the implications behind the changes they are making to the model parameters.

Alsakran et al. presented a visualization system, STREAMIT, capable of spatially arranging text streams based on keyword similarity [3]. Again, users can interactively explore and adjust the spatial layout through directly changing the weight of keywords that they find important. In addition, STREAMIT allows for users to conduct a temporal investigation of how clusters change over time.

\section{Synthesis Tools}

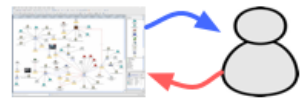

Figure 2. A model of interaction with synthesis tools. Users manually create a spatial layout of the information to maintain and organize their insights about the data.

Synthesis tools focus on allowing users to organize and maintain their hypotheses and insight regarding the data in a spatial medium. In large part, this is done through presenting users with a flexible spatial workspace in which they can organize information through creating spatial structures, such as clusters, timelines, stories, etc. (Figure 2 ). In doing so, users externalize their thought processes (as well as their insights) into a spatial layout of the information.

For example, Analyst's Notebook [2] provides users with a spatial workspace where information can be organized, and connections between specific pieces of information (e.g., entities, documents, events, etc.) can be created. Similarly, The Sandbox [26] enables users to create a series of cases (collections of information) which can be organized spatially within the workspace.

From previous studies, we found cognitive advantages associated with the manual creation of a spatial layout of the information [4]. By providing users a workspace in which to manually create spatial representations of the information, users were able to externalize their semantics of the information into the workspace. That is, they created spatial structures (e.g., clusters, timelines, etc.), and both the structures as well as the locations relative to remaining layout carried meaning to the users with regards to their sensemaking process. Marshall et al. have pointed out that 


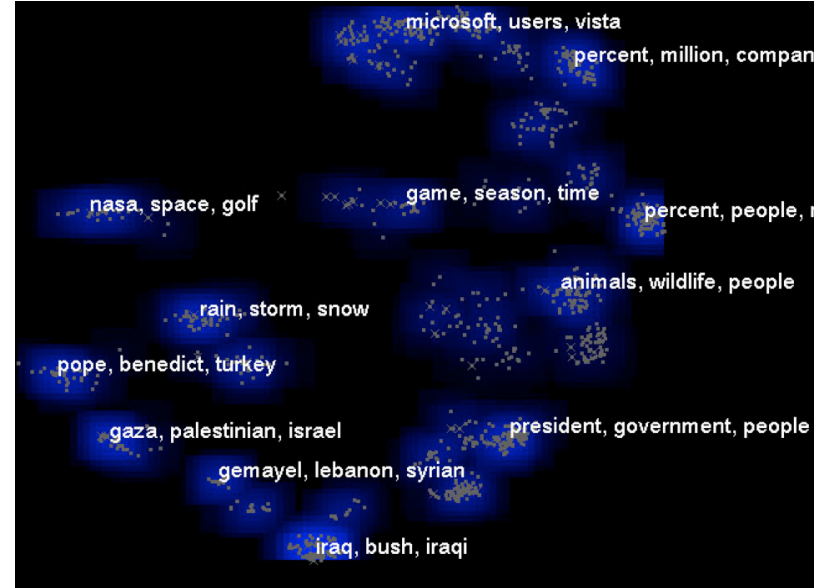

Figure 3. The IN-SPIRE Galaxy View showing a spatializtiation of documents represented as dots. Each cluster of dots represents a group of similar documents.

allowing users to create such informal relationships within information is beneficial, as it does not require users to formalize these relationships [17].

From this related work, we believe a trend is emerging in how interaction is currently handled in many visual analytic systems where complex statistical models are used - users are required to go outside of the metaphor. That is, while the visual representation given to users is spatial, the methods of interaction require users to step outside of that metaphor and interact directly with the parameters of the statistical model using visual controls, toolbars, etc.

There has been some work in providing more easy to use interactions for updating statistical models. For example, relevance feedback has been used for content-based image retrieval, where users are able to move images towards or away from a single image in order to portray pair-wise similarity or dissimilarity [24]. From there, an image retrieval algorithm determines the features and dimensions shared between the images that the user has determined as being similar. We view this as one example where the interaction stays in the spatial metaphor of the visualization.

Also, spatializations of document sets exist that allow users to place "points of interest" into the spatial layout. In VIBE, users are allowed to define multiple points of interest in the spatial layout that correspond to a series of keywords describing a subject matter of interest to the user [18]. Similarly, Dust \& Magnet [27] allows users to place a series of "magnets" representing keywords into the space and observe how documents are attracted or repelled from the locations of these magnets. Through both of these systems, users can interact in the spatial metaphor through these placements of "nodes" representing keywords. However, the focus of semantic interaction is on interacting with data (i.e., documents), an important distinction discussed in the following section.
From the sensemaking loop presented by Pirolli and Card [19], we learn that in intelligence analysis, that analytic process consists not only of the information that is explicitly within the dataset being analyzed, but also the domain knowledge of the analyst performing the analysis. It is through this domain knowledge that analysts interact and explore the dataset to "make sense" of the information. Thus, we believe this interaction (and the domain knowledge associated with it) is equally important as the raw data, and must be incorporated into the visualization by tightly coupling the model with the interaction.

From this body of work, we most notably come away with an understanding that 1) analysts fundamentally understand the spatial metaphor used in many spatial visualizations, 2) many of these systems are constructed using complex mathematical algorithms to transform high-dimensional data to two dimensions, and 3) in most cases these algorithms can be controlled by analysts largely through visual controls (e.g., sliders, knobs, etc.) to directly adjust parameters of the algorithms, updating the spatial layout.

\section{SEMANTIC INTERACTION}

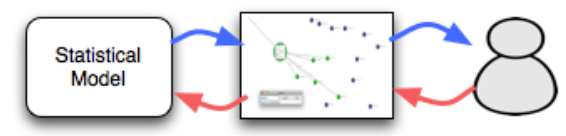

Figure 4. A model of semantic interaction. Users are able to interact directly in the spatial metaphor. The system updates the corresponding parameters of the statistical model based on the analytic reasoning of the users. Finally, the model updates the visualization based on the changes, thus unifying the synthesis and foraging stages of the sensemaking loop.

In the purest sense, semantic interaction refers to interaction occurring within a spatial visualization, with the added benefit that it is tightly coupled to the model calculating the spatial layout (Figure 4). Given the previous work of what interaction in visual analytic tools $i s$, semantic interaction occupies a new design space for interaction. It merges the ability to change the statistical model while maintaining the flexibility and familiar methods for interacting within the metaphor of spatial visualizations. Users can benefit from semantic interactions in that they can interact within a metaphor which they are familiar with, performing interactions which are part of the spatial analytic process [4], without having to focus on formal updates to the model.

Semantic interaction leverages the cognitive connection formed between the user and the spatial layout. The following intelligence analysis scenario is representative of the strategies and interactions of analysts when performing an intelligence analysis task of textual documents in a spatial visualization, as previously found by Andrews et al. [4], and further motivates and explains the concept of semantic interaction: 

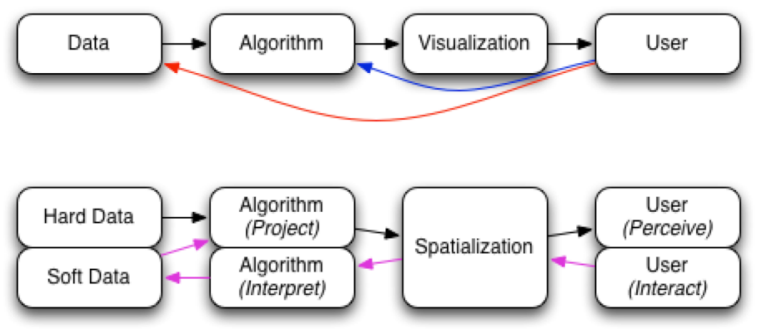

Figure 5. (top) The basic version of the "visualization pipeline". Interaction can be performed on directly the Algorithm (blue arrow) or the data (red arrow). (bottom) Our modified version of the pipeline for semantic interaction, where the user interacts within the spatial metaphor (purple arrow).

During her analysis, an intelligence analyst finds a suspicious and interesting phrase within a document. While reading through the document, she highlights the phrase "suspicious individuals were spotted at the airport", in order to more easily recall this information later. After she finishes reading the document, she moves the document into the bottom right corner of her workspace, in the proximity of other documents related to an event at an airport. To remind herself of her hypothesis, she annotates the document with "might be related to Revolution Now terrorist group". Now, with the goal of further examining the events at the "airport", she searches for the term, continuing her investigation.

In addition to the three forms of semantic interaction in the scenario, Table 1 provides a list of various forms of semantic interaction, including how each can be used within the analytic process of investigating textual information spatially. We do not claim that this list is complete, but instead point out that each of these interactions can relate to a user's reasoning within the analytic process.

\section{Designing for Semantic Interaction}

In order for analysts to interact with information in a spatial metaphor, it must first be created. Following the model of the visualization pipeline [13], this creation calls for a series of mathematical transformations, turning raw data into a spatial layout - much the way many of the visualizations mentioned previously are constructed. However, these visualizations fit this model, as their user interactions are primarily focused on directly modifying the statistical model (as well as other attributes of the visualization or data transformation). Designing for semantic interaction requires a fundamentally different model for how tools integrate user interaction - one that can capture the interaction, interpret the associated analytical reasoning, and update the appropriate mathematical parameters.

Figure 5 illustrates this model, where the spatialization is treated a medium through which the user can perceive

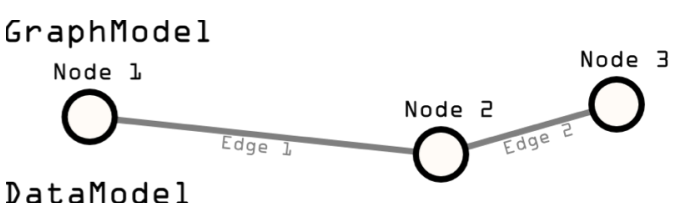

DataModel

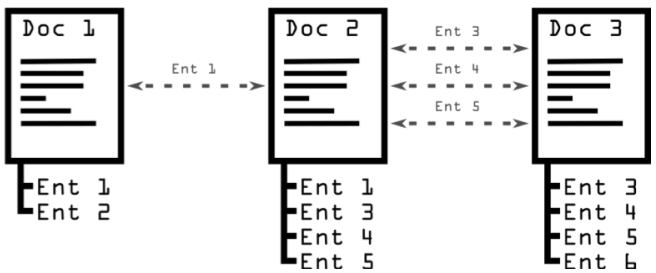

Figure 6. Overview of how nodes and edges in ForceSPIRE's force-directed layout are created from documents (Doc) and entities (Ent), respectively.

information and gain insight, as well as interact and perform his analysis. Through expanding the pipeline to accommodate for semantic interaction, it is a more appropriate match to the user's sensemaking process.

\section{Capturing the Semantic Interaction}

A non-trivial first step in the model is capturing the user interaction. Much research has been done in this area, primarily for the purpose of maintaining process history (e.g., [5], [21], [12], etc.). When considering how to capture interaction, one decision to be made is at what "level" to capture it. For example, GlassBox [6] captures interaction at a rudimentary level (i.e. mouse clicks and key strokes), while Graphical History [14] keeps track of a series of previous visualizations as a user changes the visualization during the exploration of the data.

Semantic interaction is captured at a data level, as the interactions occur on the data, and within the spatial metaphor. Using the earlier analytic scenario, the interaction being captured would be:

- The highlighted phrase

- When the highlighting occurs (timestamp)

- The color chosen for the highlight

- The document in which the highlight occurs

- The new document location

- The text of the annotation

By capturing (and storing) the interaction history, we can interpret the analytical reasoning of the user. Thus, we not only capture the interaction, but also use it.

Interpreting the Associated Analytical Reasoning

In interpreting the interaction, the goal is for the system to determine the analytical reasoning associated with the interactions and update the model accordingly. From previous findings [4], we can associate analytical reasoning with forms of semantic interaction (see Table 1). It is essentially the model's task to determine why, in terms of the data, the interaction occurred. To answer this question, we do not propose that this model can accurately gauge user intent. Instead, the goal is to calculate, based on the data, 


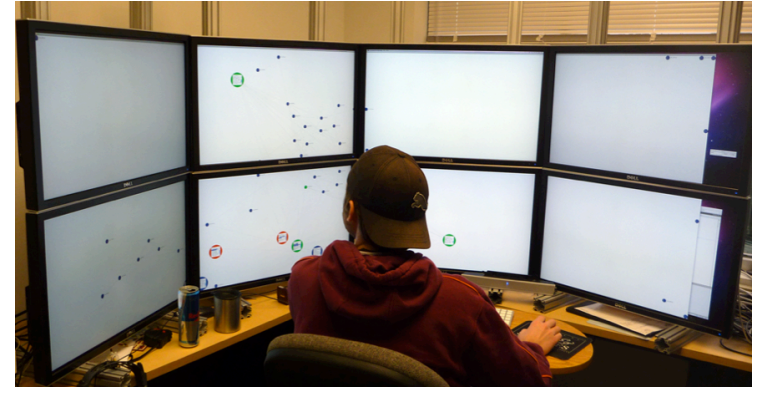

Figure 7. Using ForceSPIRE on a 32 megapixel large, high-resolution display.

what information is consistent with the captured interaction. For instance, we associate text highlighting with adding importance to the text being highlighted. We do not claim that we can associate the interaction of highlighting to the intuition that spurred the analyst to highlight the text, which is far more challenging, and arguably impossible.

We refer to the captured and interpreted interactions as soft data, in comparison to the hard data that is extracted from the raw textual information (e.g., term or entity frequency, titles, document length, etc.). We define soft data as the stored result of user interaction as interpreted by the system. In representing interaction as soft data, the algorithm can calculate and reconfigure the spatial layout accordingly. Figure 5 illustrates how our approach differs from the traditional visualization pipeline.

There has been previous work in capturing and interpreting reasoning from user interaction. For instance, Dou et al. [7] performed a study where financial analysts were asked analyze a dataset using WireVis, an interactive financial transaction visualization. The tool developers then analyzed the captured interaction, and assumptions were made about the reasoning of the analysts at specific points in the investigation. These results were compared to the analysts' self-recorded reasoning, and found to be accurate up to $82 \%$. While our work has similar goals (i.e., interpreting the analytical reasoning associated with the analysts through an evaluation of the interaction) our model does so through tightly integrating the interaction with the underlying mathematical model. In doing so, the interpretation can be done algorithmically.

\section{Updating the Underlying Model}

Through metric learning of distance weights, the layout uses the soft data to update the underlying model. Depending on the algorithm used to compute the spatial layout, the precise parameters being updated will vary. In general, this will refer to weighting of a combination of dimensions that will help guide the model as to which dimensions the user finds important.

\section{FORCESPIRE: SYSTEM OVERVIEW}

ForceSPIRE is a visual analytics prototype designed for specific forms of semantic interaction (document movement, text highlighting, search, and annotation) for
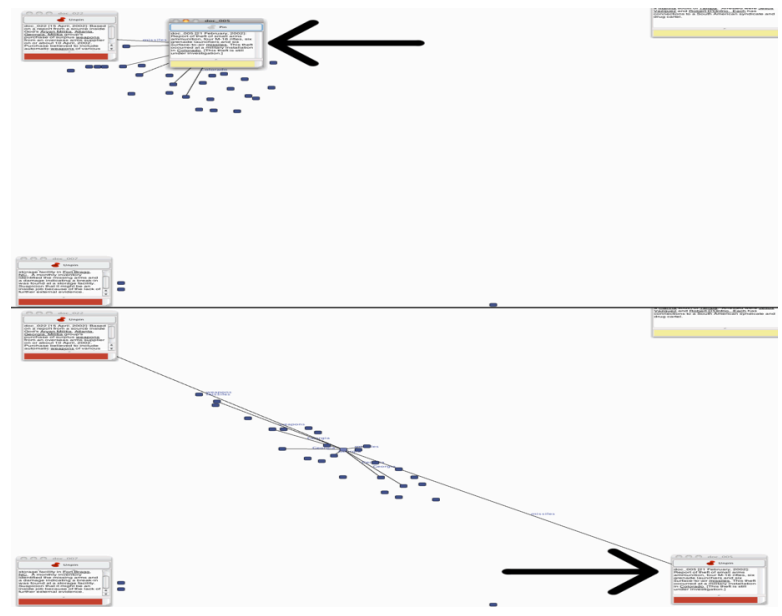

Figure 8. Moving the document shown by the arrow, ForceSPIRE adapts the layout accordingly. Documents sharing entities with the document being moved follow.

interactively exploring textual data. The system has a single spatial view (shown in Figure 12), where a collection of documents is represented spatially based on similarity (i.e., documents closer together are more similar).

ForceSPIRE is designed for large, high-resolution displays (such as the one shown in Figure 7). As semantic interaction emphasizes the importance of context in which the interaction takes place (e.g., highlighting text in the context of the document), having the full detail text available in the context of the spatial layout is beneficial over having a single document viewer. Further, the physical

Table 1. Forms of semantic interaction. Each interaction corresponds to reasoning of users within the analytic process.

\begin{tabular}{|c|c|}
\hline $\begin{array}{l}\text { Form of Semantic } \\
\text { Interaction }\end{array}$ & Associated Analytic Reasoning \\
\hline Document Movement & $\begin{array}{l}\text { - Similarity/Dissimilarity } \\
\text { - Create spatial construct (.e.g } \\
\text { timeline, list, story, etc) } \\
\text { - Test hypothesis, see how } \\
\text { document "fits" in region }\end{array}$ \\
\hline Text Highlighting & $\begin{array}{l}\text { - Mark importance of phrase } \\
\text { (collection of entities) } \\
\text { - Augment visual appearance of } \\
\text { document for reference }\end{array}$ \\
\hline $\begin{array}{lll}\text { Pinning } & \text { Document } & \text { to } \\
\text { Location } & & \end{array}$ & $\begin{array}{l}\text { - Give semantic meaning to } \\
\text { space/layout }\end{array}$ \\
\hline Annotation, "Sticky Note" & $\begin{array}{l}\text { - Put semantic information in } \\
\text { workspace, within context }\end{array}$ \\
\hline Document Coloring & $\begin{array}{l}\text { - Create visual group/cluster } \\
\text { - Mark group membership }\end{array}$ \\
\hline Level of Visual Detail & $\begin{array}{l}\text { - Change ease of visually } \\
\text { referencing information (e.g. full } \\
\text { detail = more important = easy to } \\
\text { reference) }\end{array}$ \\
\hline Query Terms & - Expressive search for entity \\
\hline
\end{tabular}




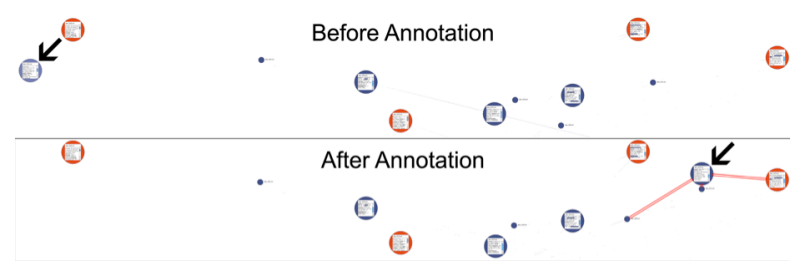

Figure 9. The Effect of adding an annotation ("these individuals may be related to Revolution Now") to the document shown with an arrow. As a result, the document becomes linked with other documents mentioning the terrorist organization "Revolution Now".

presence of these displays creates an environment in which the virtual information (in this case the documents) can occupy persistent physical space. As a result, users are further immersed into the spatial metaphor, as they can point and quickly refer to information based on the physical locations.

\section{Constructing the Spatial Metaphor}

The spatial layout of the text documents is determined by a modified version a force-directed graph model [11]. This model functions on the principle of nodes with a mass connected by springs with varying strengths. Thus, each node has attributes of attraction and repulsion: nodes repel other nodes, and two nodes attract each other only when connected by a spring (edge). The optimal layout is then computed by iteratively calculating these forces until the lowest energy state of all the nodes is reached. A complete description of this algorithm can be found in [11].

We apply this model to textual information by treating documents as nodes (an overview is shown in Figure 6). The entire textual content of each document is parsed into a collection of entities (i.e., keywords). The number of entities corresponds to the mass of each document (heavier nodes do not move as fast as lighter nodes). A spring (or edge) represents one or more matching entities between two nodes. Therefore, the initial distance metric is a based on co-occurrence of terms between documents. For example, two documents containing the term "airport" will be connected by a spring. The strength of a spring (i.e. how close together it tries to place two nodes) is based on two factors: the number of entities two documents have in common, and the importance value associated with each shared entity (initially, importance values are created using a standard tfidf method [16]). The sum of all importance values add up to 1 .

The resulting spatial layout is one where similarity between documents is represented by distance relative to other documents. Similarity in this system is defined by the strength of the spring between two documents. A stronger spring (and therefore a larger amount of shared entities) will pull two documents closer together, and thus represent two similar documents.

\section{Semantic Interaction in ForceSPIRE}

The semantic interactions in ForceSPIRE are: placing information at specific locations, highlighting, searching, and annotating in order to incrementally change the spatial layout to match their mental model. The primary parameters of the force-directed model that are being updated through this learning model are the importance values of the entities.

Document Movement. The predominant interaction in a spatial workspace is positioning (and repositioning) documents. In previous work, we have demonstrated how users can perform both exploratory and expressive forms of this type of interaction [9]. In ForceSPIRE, we allow for the following exploratory interaction (i.e., interaction that allows users to explore the structure of the current model, but does not change it). Users are able to interactively explore the information by dragging a document within the workspace, pinning a document to a particular location (see Figure 8), as well as linking two documents. When dragging a document, the force-directed system responds by finding the lowest energy state of the remaining documents given the current location of the dragged document. Mathematically, this adds a constraint to the stress function being optimized (in this case the force-directed model). This allows users to explore the relationship of that document in comparison to the remaining documents.

In addition to the exploratory dragging of a document, users have the ability to pin a document. By pinning a document, users are able to incrementally add semantic meaning to locations in their workspace. By specifying key documents to user-defined locations, the layout of the remaining documents will adapt to these constraints. Thus, users can explore how documents are positioned based on their similarity (or dissimilarity) to the pinned documents. For instance, if the layout places a document between two pinned documents, it may imply that the particular document holds a link between the two pinned documents, sharing entities that occur in both.

Finally, users can perform an expressive form of this interaction by linking two documents, performed by dragging one document onto another pinned document. In doing so, ForceSPIRE calculates the similarity between the documents, and increases the importance value of the entities shared between both documents. As a result, the layout will place more emphasis on the characteristics that make those two documents similar.

Highlighting. When highlighting a term, ForceSPIRE creates an entity from the term (if not already one), and the importance value of that term is increased. Similarly, highlighting a phrase results in the phrase being first parsed for entities, then increasing the importance value of each of those entities. For example, Figure 11 shows the effect of highlighting the terms "Colorado" and "missiles" in the document pointed to with the arrow. As a result, the 


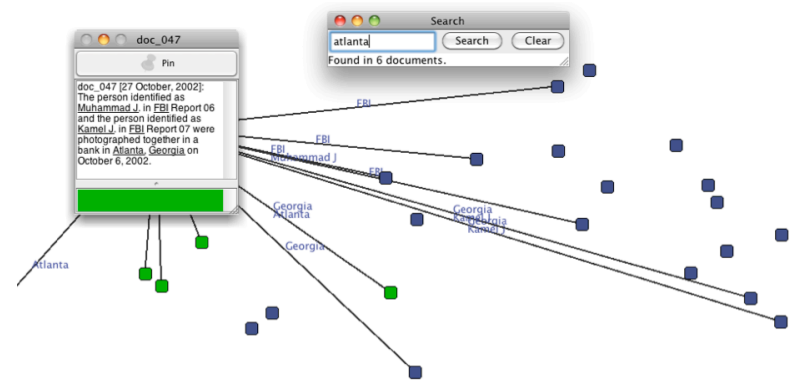

Figure 10. Searching for the term "Atlanta", documents containing the term highlight green within the context of the spatial layout. Additionally, the importance value of entity "Atlanta" is increased.

other documents containing that term are clustered more tightly.

Searching. When coming across a term of particular interest, analysts usually search on that term in order to find other occurrences. In a spatial workspace, this is of particular importance, because the answer to "where the term is also found" is not only given in terms of what documents, but also where in the layout those documents occur. The positions of documents containing the term are shown in context of the entire dataset, from which users can infer the importance of that term (as shown in Figure 10).

ForceSPIRE first creates an entity from the search term (unless it is already one), then increases the importance value of the search term. Figure 10 gives an example of how a search result appears in ForceSPIRE. Searching for the term "Atlanta", documents that contain the term are highlighted green, and links are drawn to show where the resulting documents are in relation to the current document.

Annotation. Annotations (i.e., "sticky notes") are also viewed as a form of semantic interaction, occurring within the analytic process, from which analytic reasoning can be inferred. When a user creates a note regarding a document, that semantic information should be added to the document. For example, if Document A refers to "Revolution Now" (a suspicious terrorist group), and Document B refers to "a group of suspicious individuals", and the user has reason to believe these individuals are related to Revolution Now, adding a note to Document B stating "these individuals may be related to Revolution Now" is one way for the user to add semantic meaning to the document.

ForceSPIRE handles the addition of the note (shown in Figure 9) by 1) parsing the note for any currently existing entities, then 2) increasing the importance value of each, and 3 ) creating any new springs between other documents sharing these entities. In the example in Figure 9, edges are created between Document B and Document A (as well as any other documents that mention "Revolution Now"). Additionally, if the note contains any new entities not currently in the model, they are created, with the intent that

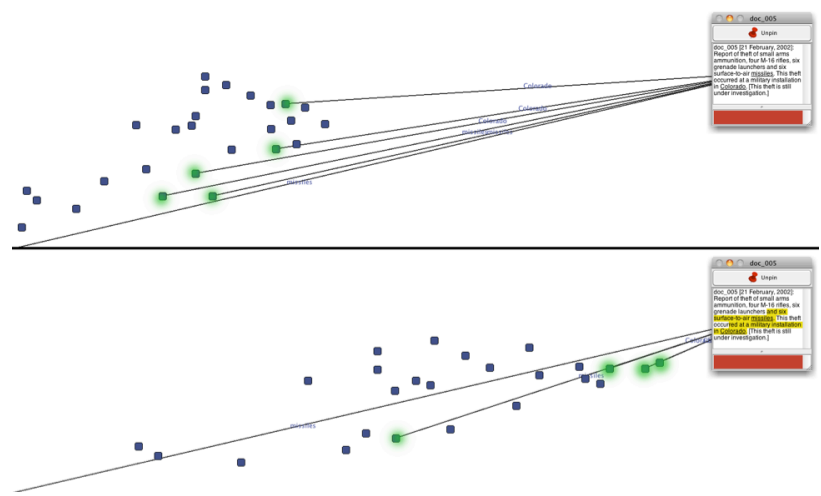

Figure 11. The effect of highlighting a phrase containing the entites "Colorado" and "missiles". Documents containing these entities move closer, as the increase in importance value increases the edge strength.

any future entities that may match to that note can be linked at that time. ForceSPIRE also handles cases where notes are edited, with text added or removed from the note, by updating the entities associated with the document, and adjusting the importance values of these entities accordingly.

\section{Model Updates}

Each of the semantic interactions in ForceSPIRE impacts the model by updating the importance values of entities, and the mass of each document. The calculation for updating the importance value of an entity is the same for each interaction. If an entity was "hit" (i.e., it was included in a highlight, it was searched, it was in a note, etc.), ForceSPIRE increases its importance value by $10 \%$. As the sum of all importance values of entities adds up to 1, ForceSPIRE subtracts an equal amount from all other entities' importance values. As a result, importance values decay over time, and entities that are rarely used during the analysis have less impact on the layout. The mass of a document uses a similar calculation, in that each time a document is "hit" (i.e., text was highlighted, it was the result of a search hit, etc.), it increases by $10 \%$.

When undoing an interaction using the standard "Control+Z" keyboard shortcut, a linear history of the interactions will be reversed, and the importance values of affected entities will be returned to their prior values (as well as document masses). As for the locations of the documents, the reverted importance values and document masses will be responsible for updating the layout. However, this does not guarantee that the layout will return to the exact previous view, and the user may find it necessary to perform small adjustments.

The model updates used in ForceSPIRE serve as an initial approach at how to couple semantic interactions with model updates. Other, more complex methods may exist, and we encourage further research in this area. Sensemaking is a complex exploratory process. As such, semantic interaction 


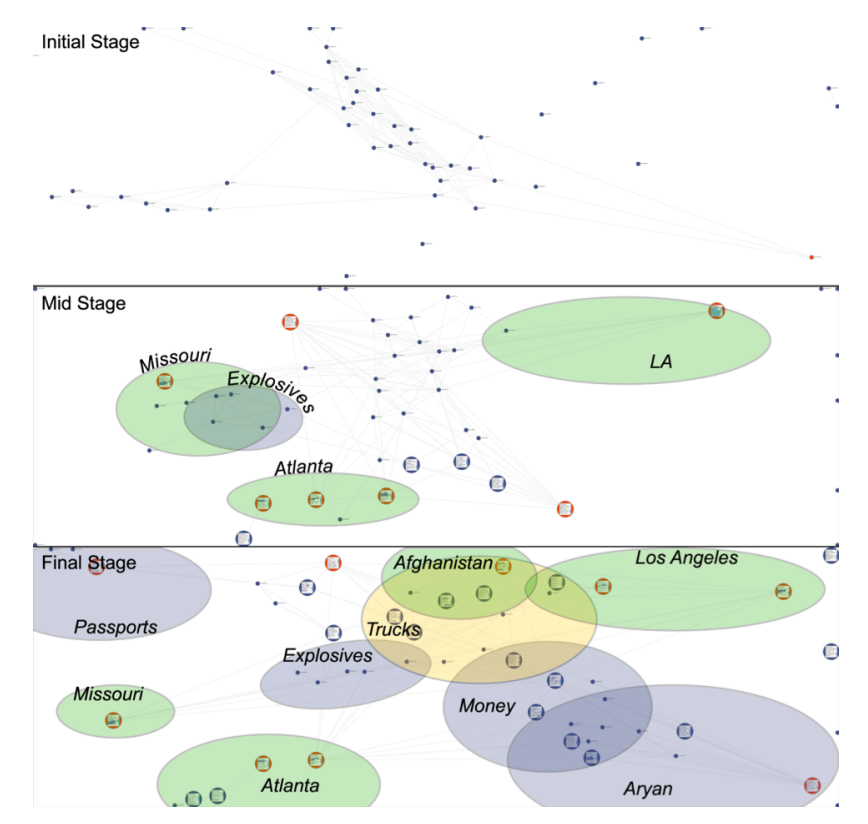

Figure 12. The incremental change of the spatial layout (main view of ForceSPIRE) from the initial to the final state. Through semantic interaction, the layout incrementally changed based on the semantic input of the user. We labeled the regions based on what the user told us the regions meant to him at each stage.

can enable analysts to explore their hypothesis in-situ, while the provenance of their insights is captured and stored. An open area of research is what analyzing the soft data might reveal about the analytic process. For instance, if the importance values of entities converge on a small number of entities, specific biases might be revealed. Similarly, instances during the analysis when new hypotheses are being explored may be indicated by diverging importance values.

\section{Use Case}

We demonstrate the functionality of ForceSPIRE through the following use case. In this scenario, we simulate an intelligence analysis scenario where the task is to find a hidden terrorist plot in a pre-constructed, ficticious textual dataset. The dataset consists of 50 text documents, containing a complex terrorist plot (explosives are being transported to various cities in the U.S. using trucks). The combination of the task of finding the hidden terrorist plot and the textual dataset is representative of daily work performed by professional intelligence analysts [8]. The analysis described below lasted 70 minutes, and was performed by an individual computer science graduate student.

The user began the investigation by loading the collection of documents into ForceSPIRE. The documents were automatically parsed for entities using the LingPipe keyword extraction library [1]. From these entities, an initial layout was generated, shown in Figure 12(top). From this layout, he began investigation by reading through the more central documents. While reading through the documents, he highlighted phrases of interest. For example, he highlighted the phrase "Nizar A. is now known to have spent six months in Afghanistan". In doing so, ForceSPIRE increased the importance value of the entities within the phrase, particularly "Afghanistan" and "Nizar A". As a result, the layout forms more tightly around those entities. Each change incrementally changes the layout.

Continuing with his investigation, he began searching for words of interest (e.g., "weapons", "Colorado", "Atlanta", etc.). ForceSPIRE provided him with quick visual feedback on where in the dataset each terms showed up (the search result for "Atlanta" is shown in Figure 10). In addition to gaining an overview of the distribution of the term within the dataset (by highlighting each document containing the term green), ForceSPIRE treats performing a search as either creating a new entity from the search term, or increasing the importance value if an entity corresponding to the search term already exists. As a result of the multiple search terms and highlights corresponding to locations (e.g., "Atlanta", "Los Angeles", "Missouri", etc.), ForceSPIRE adapts the spatialization by creating a more geographicoriented layout (shown in the "Mid Stage" layout in Figure 12).

During further investigation, he began opening more documents and adding annotations to documents where he found information missing that he knew. For example, Figure 9 shows how he opened one document where "suspicious individuals" were mentioned. Earlier, he read a document containing information about a terrorist organization named "Revolution Now". While reading about the suspicious individuals, the other information in the document triggered him to make a connection between these individuals and Revolution Now. He made added a note to the document about the suspicious individuals stating "these individuals may be related to Revolution Now". As a result, ForceSPIRE parsed the note for entities, added them to the document, and pulled the document closer to other documents containing the entity "Revolution Now".

After continuing his investigation in this manner, he ultimately made the connections within the dataset to uncover the terrorist plot. The progression of the spatial layout, shown in Figure 12, shows the final layout, where he was able to pinpoint regions of the layout as being important in his finding. Some of the spatial locations of clusters are a result of him pinning documents to that region (e.g., "Atlanta", "Los Angeles", etc.). These pinned documents are shown in red. Perhaps more interestingly is not the regions that were created as a result of him pinning documents to that location, but rather how the remaining documents respond in the layout. For example, in the final state shown in Figure 12, a group of documents began to emerge in the middle of all the pinned locations. Upon examining these documents, he discovered that these 


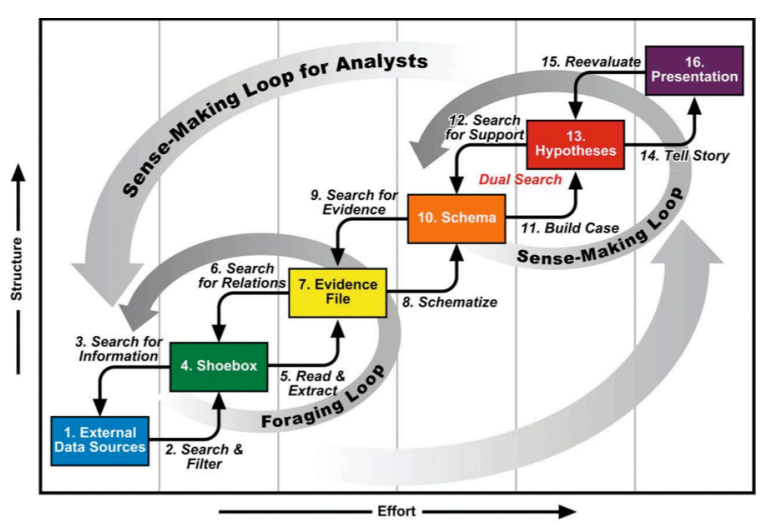

Figure 13. The sensemaking loop, illustrating the complex sequence of steps used by intelligence analysts in order to gain insight into data.

documents are about the terrorist organization using "UHaul" or "Ryder" trucks for transportation between these locations. ForceSPIRE placing these documents in between these cities in the layout was helpful, as these documents contain information "connecting" the events in these locations. Immediately after noticing this event, he also made use of the expressive form of interaction, performed by dragging two of these documents together to determine what made them similar. After seeing that it was indeed terms such as "Ryder" and "U-Haul", the layout formed more tightly around these terms.

ForceSPIRE interpreted the analytical reasoning of the user through the creation of new entities that were not found by the initial keyword extraction, as well as the increase of importance values of existing entities. This is evidenced by the creation of 39 new entities during the course of the analysis. LingPipe extracted 89 initial entities from this dataset, and at the time of completing our investigation ForceSPIRE included 128. Examples of newly created entities are "big event", "grenades", "Fisher Island", "weapons", and others. The ability for new entities to be created via semantic interaction did not interfere with the fluid sensemaking process of the user. Instead, it aided the process by creating new entities, which in turn created semantically relevant connections within the dataset.

In addition to creating new entities, existing entities dynamically changed their importance value based on the interpreted analytical reasoning of the semantic interactions. Examples of entities that changed their importance values are "Atlanta", "Revolution Now", "Colorado", "L.A.", and others. As a result, the ForceSPIRE incrementally adapted the layout based on the user input. This shows that adjusting importance values, creating entities, and changing locations of key documents helped the user discover the structure of the dataset, and ultimately make out the hidden terrorist plot.

\section{DISCUSSION}

\section{Unifying the Sensemaking Loop}

With the fundamentally different role occupied by semantic interaction, we explore a new design space for interaction in visual analytic tools. With the addition of soft data, and a model capable of interpreting the user's analytical reasoning, we leverage interactions that are already occurring in the spatial analytic process to further aid users in their sensemaking process.

With semantic interaction, the amount of formalization between foraging and sensemaking (Figure 13) on the part of the user is reduced. For instance, in moving a document, users can formulate a hypothesis based on that document, expecting similar documents to follow. ForceSPIRE attempts to update the layout based on the interaction, and gives the user feedback. Thus, the foraging stage occurs as a result of the hypothesis being formed through semantic interaction. By not forcing users to over-formalize their analytic reasoning too early in order to forage for the relevant information, semantic interaction creates a more seamless transition between foraging and synthesis, unifying the sensemaking loop.

\section{Future Work}

Semantic interaction, as a concept, opens up many possibilities for further research, such as: what interactions to capture and store, which parameters of the model to update, how to store the soft data, and which models present a metaphor that can be extended upon.

In order to make more concrete claims regarding the usability and effectiveness of ForceSPIRE (and thus, of semantic interaction), a formal user study is needed. Our plan is to introduce ForceSPIRE to professional intelligence analysts and have them solve scenarios that model their daily task, such as one of the VAST datasets [2020]. The observations and feedback from these users will provide ecological validity for semantic interaction.

\section{CONCLUSION}

In this paper we have discussed how the concept of semantic interaction leads to a new design space for interaction in spatializations of textual information. Semantic interactions occur directly within the spatial metaphor, support spatial cognition, and exploit spatial analytic interactions. We describe semantic interaction, discussing the three components required - capturing the interaction, interpreting the analytical reasoning, and updating the mathematical model. Further, we present ForceSPIRE, designed for semantic interaction with textual information, discussing its functionality and demonstrating how it can be used through a use case. Lastly, we discuss how semantic interaction has the opportunity to unify the sensemaking loop, creating a more seamless analytic process. In allowing users to interact within the spatial metaphor, they can remain more focused on their analysis of the data, without having to become experts in the underlying mathematical models of the system. 


\section{ACKNOWLEDGEMENTS}

This research was funded by the NSF grant CCF-0937071 and the DHS center of excellence.

\section{REFERENCES}

1. Alias-i. 2008. LingPipe 4.0.1. City, 2008.

2. i2 Analyst's Notebook. City.

3. Alsakran, J., Chen, Y., Zhao, Y., Yang, J. and Luo, D. STREAMIT: Dynamic visualization and interactive exploration of text streams. In Proceedings of the IEEE Pacific Visualization Symposium, 2011.

4. Andrews, C., Endert, A. and North, C. Space to Think: Large, High-Resolution Displays for Sensemaking. In Proceedings of the CHI '10, 2010.

5. Callahan, S. P., Freire, J., Santos, E., Scheidegger, C. E., C, Silva, u. T. and Vo, H. T. VisTrails: visualization meets data management. In Proceedings of the SIGMOD international conference on Management of data (Chicago, IL, USA, 2006). ACM.

6. Cowley, P., Haack, J., Littlefield, R. and Hampson, E. Glass box: capturing, archiving, and retrieving workstation activities. In Proceedings of the workshop on Continuous archival and retrival of personal experences (Santa Barbara, California, USA, 2006). ACM.

7. Dou, W., Jeong, D. H., Stukes, F., Ribarsky, W., Lipford, H. R. and Chang, R. Recovering Reasoning Processes from User Interactions. IEEE Computer Graphics and Applications, 2009.

8. Endert, A., Andrews, C., Fink, G. A. and North, C. Professional Analysts using a Large, High-Resolution Display. In Proceedings of the IEEE VAST Extended Abstract (2009).

9. Endert, A., Han, C., Maiti, D., House, L., Leman, S. C. and North, C. Observation-level Interaction with Statistical Models for Visual Analytics. IEEE VAST, 2011.

10. Frank M. Shipman, I. and Marshall, C. C. Formality Considered Harmful: Experiences, Emerging Themes, and Directions on the Use of Formal Representations inInteractive Systems. ACM CSCW, 8, 4, 1999, 333-352.

11. Fruchterman, T. M. J. and Reingold, E. M. Graph drawing by force-directed placement. Software: Practice and Experience, 21, 11 1991, 1129-1164.

12. Gotz, D. Interactive Visual Synthesis of Analytic Knowledge. IEEE VAST, 2006.

13. Heer, J. prefuse manual, 2006.

14. Heer, J., Mackinlay, J., Stolte, C. and Agrawala, M. Graphical Histories for Visualization: Supporting Analysis, Communication, and Evaluation. IEEE Transactions on Visualization and Computer Graphics, 14, 6 , 2008, 1189-1196.
15.Jeong, D. H., Ziemkiewicz, C., Fisher, B., Ribarsky, W. and Chang, R. iPCA: An Interactive System for PCAbased Visual Analytics. Computer Graphics Forum, 28, 2009, 767-774.

16. Karen A Statistical Interpretation of Term Specificity and its Application in Retrieval. Journal of Documentation, 28, 1972, 11-21.

17. Marshall, C. C., Frank M. Shipman, I. and Coombs, J. H. VIKI: spatial hypertext supporting emergent structure. In Proceedings of the European conference on Hypermedia technology (Edinburgh, Scotland, 1994). ACM.

18. Olsen, K. A., Korfhage, R. R., Sochats, K. M., Spring, M. B. and Williams, J. G. Visualization of a document collection: the vibe system. Information Process Management, 29, 1 1993, 69-81.

19. Pirolli, P. and Card, S. Sensemaking Processes of Intelligence Analysts and Possible Leverage Points as Identified Though Cognitive Task Analysis Proceedings of the International Conference on Intelligence Analysis, 2005, 6.

20. Plaisant, C., Grinstein, G., Scholtz, J., Whiting, M., O'Connell, T., Laskowski, S., Chien, L., Tat, A., Wright, W., Gorg, C., Zhicheng, L., Parekh, N., Singhal, K. and Stasko, J. Evaluating Visual Analytics at the 2007 VAST Symposium Contest. Computer Graphics and Applications, IEEE, 28, 2 2008, 12-21.

21. Shrinivasan, Y. B. and Wijk, J. J. v. Supporting the analytical reasoning process in information visualization. In Proceedings of the CHI '08 (Florence, Italy, 2008). ACM.

22. Skupin, A. A Cartographic Approach to Visualizing Conference Abstracts. IEEE Computer Graphics and Applications, pp. 50-58, January/February, 2002.

23. Thomas, J. J., Cook, K. A., National, V. and Analytics, C. Illuminating the path. IEEE Computer Society, 2005.

24. Torres, R. S., Silva, C. G., Medeiros, C. B. and Rocha, H. V. Visual structures for image browsing. In Proceedings of the conference on Information and knowledge management (New Orleans, LA, USA, 2003). ACM.

25. Wise, J. A., Thomas, J. J., Pennock, K., Lantrip, D., Pottier, M., Schur, A. and Crow, V. Visualizing the nonvisual: spatial analysis and interaction with information for text documents. Morgan Kaufmann Publishers, 1999.

26. Wright, W., Schroh, D., Proulx, P., Skaburskis, A. and Cort, B. The Sandbox for analysis: concepts and methods. In Proceedings of the CHI '06 (New York, NY, 2006). ACM.

27. Yi, J. S., Melton, R., Stasko, J. and Jacko, J. A. Dust \& magnet: multivariate information visualization using a magnet metaphor. Information Visualization, 4, 4, 2005, 239-256. 